\title{
His bundle recording and electrical stimulation of atria in patients with Wolff-Parkinson-White syndrome type A
}

\author{
P. Touboul, Y. Tessier, ${ }^{1}$ J. Magriña, C. Clément, and J. P. Delahaye \\ From Hopital cardio-vasculaire et pneumologique, 59 boulevard Pinel, 69 Lyon $3^{e}$, France
}

Two patients presenting with WPW syndrome type $A$ were studied by His bundle potential recording, endocavity electrocardiogram, and electrical stimulation of right and left cardiac cavities.

The beginning of the ventriculogram preceded the appearance of the His bundle potential confirming the pre-excitation of the ventricles by another conduction system than the normal one.

Increasing the prematurity of coupled right atrial extrasystoles increased the degree of delay on the normal pathway and therefore increased the degree of pre-excitation. Both pathways

' contributed to ventricular depolarization, as shown by an increasingly longer total depolarization time.

The pre-excitation was more obvious with left atrial stimulation. Ventricular depolarization - seemed to be completely taken in charge by the anomalous pathway allowing no time for excitation from normal pathway as shown by a constant total depolarization time. In one patient, the atriobundle of His conduction was shorter with left atrial than right atrial stimulation.

During bouts of tachycardia, which were more easily induced from the left cavities than from the right, retrograde conduction was faster to the left than to the right atrium.

Our findings are consistent with an anomalous pathway lying preferentially on the left side of the heart in WPW syndrome type $A$.

The present conception of the Wolff-Parkinson-White (WPW) syndrome depends on the existence of accessory atrioventricular (AV) fibres short-circuiting the normal nodal pathway. This concept has been reinforced by new methods of cardiac exploration developed during recent years. Thus the recording - of the potential of the His bundle by cardiac catheterization has confirmed the reality of the short circuit of the His bundle in WPW syndrome (Castellanos et al., 1970; Castillo and Castellanos, 1970; Coumel et al., 1970; Touboul et al., 1971). Electrical stimulation of the heart has also enabled a better understanding of the mechanism of the pre-excitation (Lau et al., 1967; Durrer et al., 1967; Puech et al., I968). Recently Wellens, Schuilenberg, and Durrer (197I) have reported the results of an investigation of WPW syndrome type A by left atrial stimulation. These authors have found strong arguments

${ }^{1}$ Present address: Hôpital St-Sacrement, 1050 Chemin Ste-Foy, Québec 6, P.Q., Canada.

Received 4 August 1971. in favour of a localization of the accessory pathway on the left side of the heart.

We had the opportunity of performing a study of the pre-excitation in 2 patients presenting a WPW syndrome type A using the His bundle recording and the stimulation of the left atrium and the right atrium.

\section{Patients and methods}

In the Table are presented the sex, ages, types of WPW syndrome, and modalities of the tachycardia of the two patients studied. Electrocardiograms are presented in Fig. $I$ and 2. They fulfil the criteria of WPW syndrome type $A$ in Case $I$

TABLE Summary of patients' data

\begin{tabular}{lllll}
\hline Case No. & Sex & Age $(y r)$ & WPW (type) & Modalities of tachycardia \\
\hline I & F & 37 & A & $\begin{array}{c}\text { Paroxysmal atrial fibrillation and } \\
\text { regular tachycardia intermittently } \\
\text { for a week }\end{array}$ \\
2 & F & 60 & A with LAD & 'Palpitations' for six years \\
\hline
\end{tabular}


FIG. I Case I. WPW syndrome type $A$.

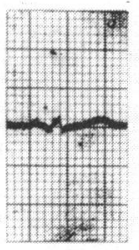

I
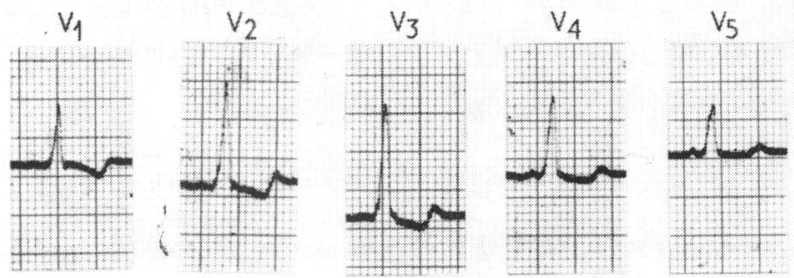

aVL

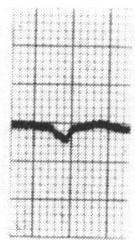

oVF
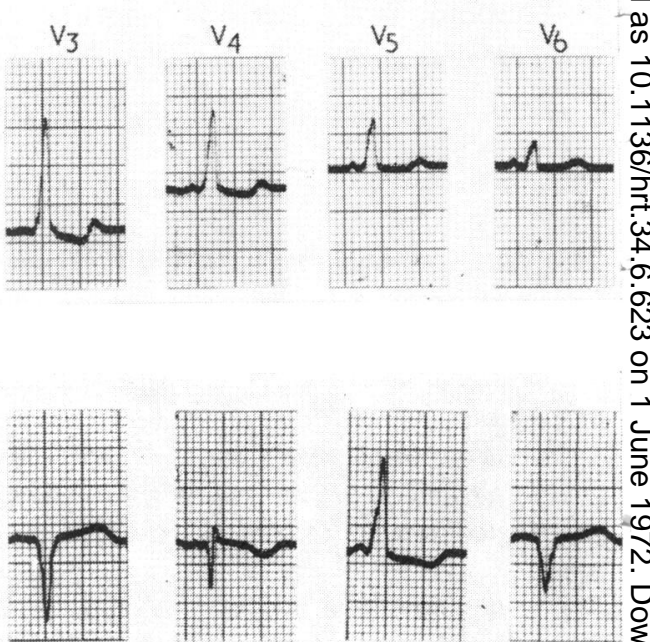

III

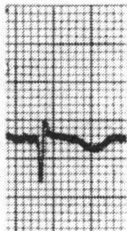

aVR

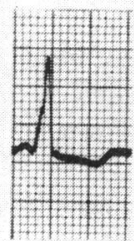

OVL

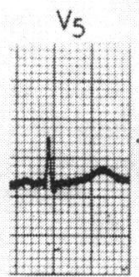

$\underline{\underline{\underline{T}}}$

T⿱亠乂

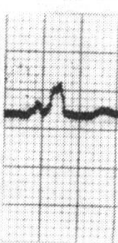

ని

음

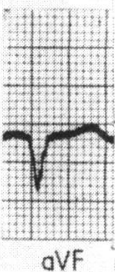

aVF

$v_{6}$

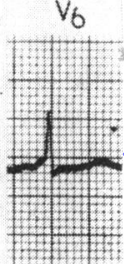

FIG. 3 Case I. Coupled right atrial stimulation. To read the ordinate, add loo for $\mathrm{SH}^{-} \mathrm{SR}^{-}$ and 200 for SY-. See terminology for the definitions of the different intervals. Shortening the $P S$ increases the $\mathrm{SH}^{-}$interval and in parallel the $S f^{-} . S R^{-}$is constant. With very short PS (250 msec) there is an intra-atrial delay (longer $\mathrm{SP}^{-}$) responsible for the lengthening of the other intervals.
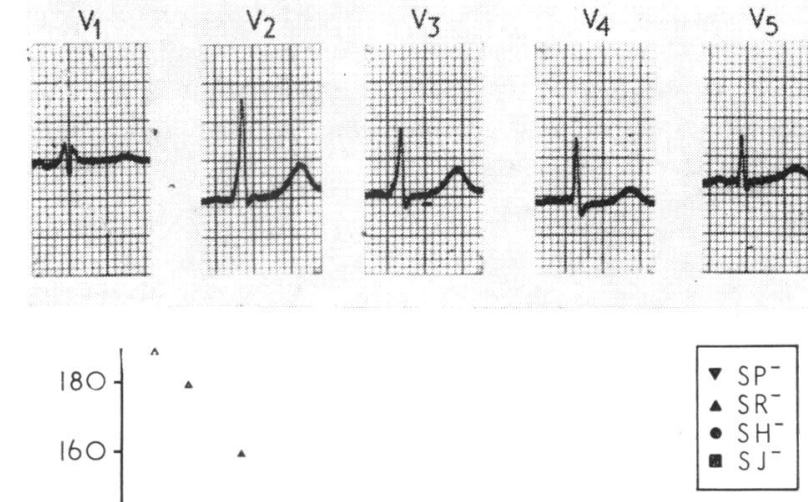

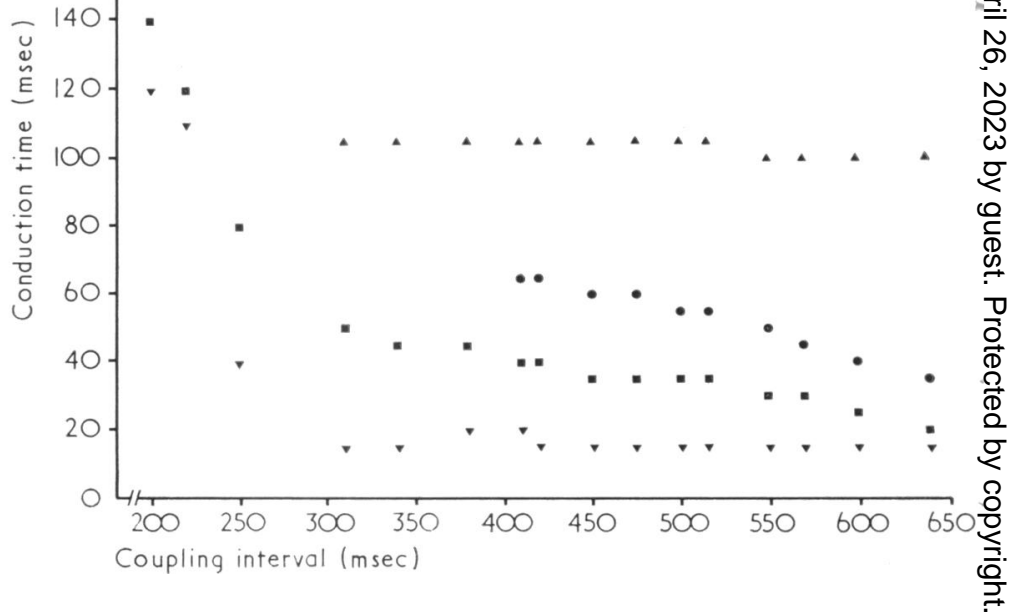


and type $A$ with left axis deviation (LAD) in Case 2, according to Rosenbaum, Hecht, and Wilson (1945).

Patients were premedicated with pentobarbitone $120 \mathrm{mg}$ in suppository form. His bundle recording was performed according to the methods of Scherlag et al. (1969). Under local anaesthesia, a bipolar catheter ${ }^{1}$ (distance between the electrodes: Io $\mathrm{mm}$ ) was introduced by the Seldinger technique through the right femoral vein and fluoroscopically positioned across the septal leaflet of the tricuspid valve where His bundle activity $(\mathrm{H})$ could be recorded as a biphasic or triphasic deflection of 15 to $20 \mathrm{msec}$ duration. Using the same technique in the left femoral vein, a multipolar catheter ${ }^{2}$ ( 5 pairs of electrodes: $2 \mathrm{~mm}$ distance between the electrodes and $10 \mathrm{~mm}$ between the pairs) was positioned in the right atrium. Later it was manoeuvred across an open foramen ovale in the left atrium. The tip electrodes were used to deliver the electrical stimuli and the next pair of electrodes to record the potentials. A careful sketch of the positions of the electrodes was done: in the right atrium the tip electrodes were in contact with the sinus node region and the other electrodes near the external border of the right atrium. In the left atrium the tip lay near the atrial appendage and the proximal electrodes curved in the superior third of the atrium.

The different leads were connected to a 4-channel direct ink recorder ${ }^{3}$ via an incorporated distribution switch box: filtering and preamplification were applied to the His lead derivation. Paper speeds were 50 or $100 \mathrm{~mm} / \mathrm{sec}$. Rapid atrial pacing was done using a Medtronic model 584I pacemaker. The stimuli were $2 \mathrm{msec}$ duration at twice the diastolic threshold value. The coupled electrical stimulation was performed with a Medtronic model 5837 stimulator. Coupling to $P$ waves was done at decreasing intervals. When tachycardia was induced, it was stopped by an appropriately coupled stimulus.

\section{Terminology}

The different intervals were determined as follows. PS: beginning of spontaneous sinus depolarization to stimulus deflection (coupling interval in the figure). $\mathrm{SP}^{-}$: stimulus deflection to intrinsicoid deflection of the endocavitary atriogram: atrial response delay to stimulation. $\mathrm{SH}^{-}$: stimulus deflection to first rapid deflection of the His bundle activity: atrium to His conduction time. $\mathrm{SR}^{-}$: stimulus deflection to beginning of the delta wave: stimulus propagation to the ventricles. $\mathrm{SJ}^{-}$: stimulus deflection to end of the longest ventriculogram: total heart depolarization time. All intervals are expressed in milliseconds (msec).

\section{Results}

Right atrial stimulation Fig. 3 and 4 illustrate the effect of electrically induced atrial extrasystoles at decreasing PS. From

1 U.S.C.I. $56516 \mathrm{~F}$.

2 Elecath 6F.

${ }^{3}$ Elema Schönander Mingograph model EM 34.

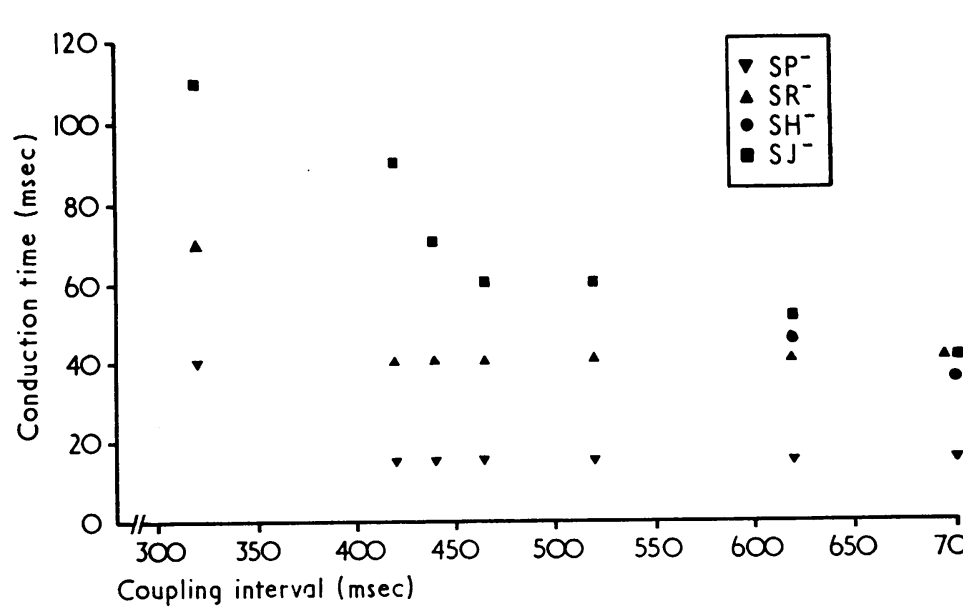

FIG. 4 Case 2. Coupled right atrial stimulation. To read the ordinate, add 100 for $\mathrm{SH}^{-}$ and 200 for $\mathrm{SF}^{-}$. With shorter PS, there are increasing $\mathrm{SH}^{-}$and $\mathrm{Sf}^{-}$intervals. $\mathrm{SR}^{-}$is constant. With PS shorter than 400 msec: intra-atrial delay. .

FIG. 5 Case 1. Coupled right atrial stimulation. In each sequence the first beat is a sinus beat, the second is electrically induced $(S)$. With shorter PS, note the progression of His potentials $\left(\mathrm{H}^{-}\right)$into the $Q R S$ and the parallel $S f^{-}$lengthening.

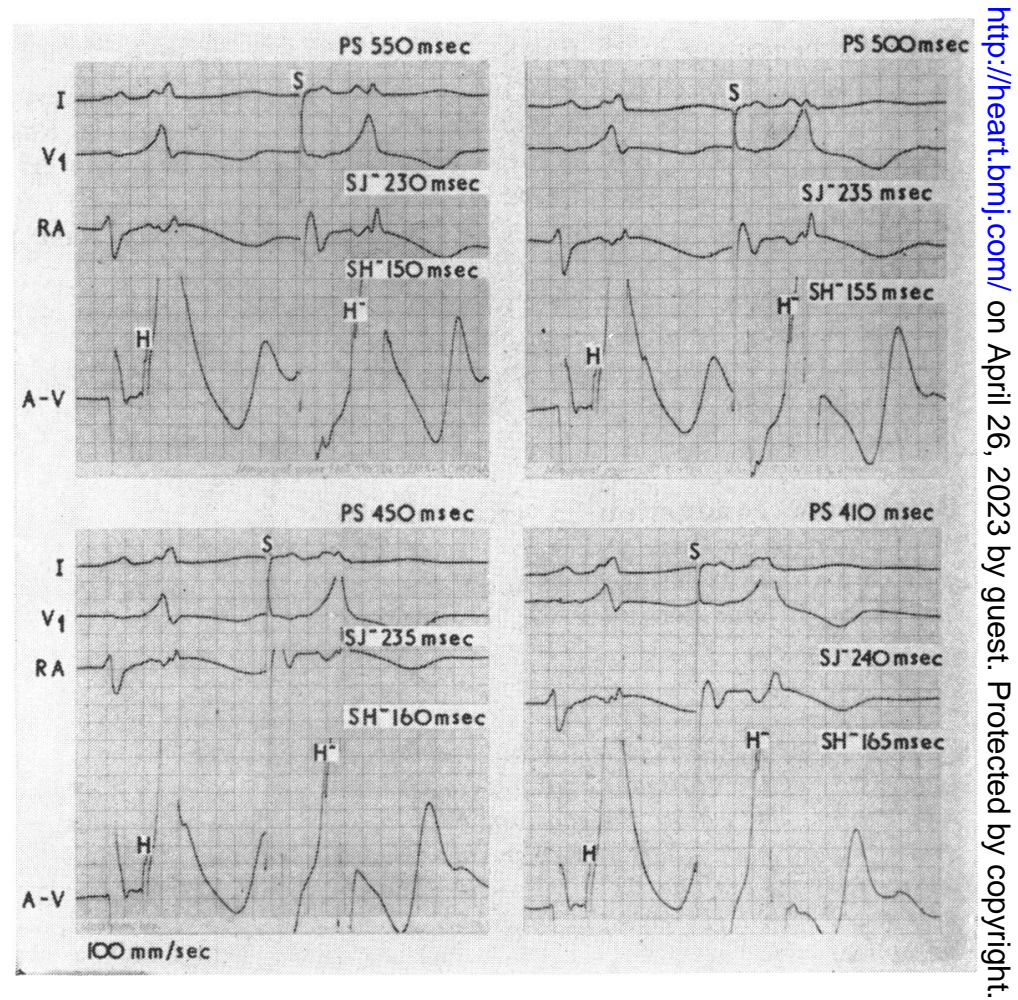


PS of $640 \mathrm{msec}$ to PS of $310 \mathrm{msec}$ in Case I and from PS of $700 \mathrm{msec}$ to PS of $420 \mathrm{msec}$ in Case 2, there is a constant $\mathrm{SR}^{-}$interval (I05 and I40 msec, respectively). Conduction from stimulus to beginning of ventricular depolarization is therefore constant. With further shortening of the coupling interval, SRincreases: this is due to an intra-atrial delay as shown by a roughly parallel increase of $\mathrm{SP}^{-}$. On the other hand, there seems to be a relation between the increasing delay of the $\mathrm{SH}^{-}$and the increasing total depolarization time. In the actual tracing of Case I shown in Fig. 5, the His deflection is after the beginning of QRS. With a shorter PS, His deflection progresses in the QRS. In addition, as compared to sinus beats, the pre-excitation appearance is augmented. In Case 2, rapid right atrial stimulation (Fig. 6) induces the same effects as coupled RA stimulation: lengthening of $\mathrm{SJ}^{-}$and constant $\mathrm{SR}^{-}$. LA stimulation is shown for comparison (see later results).

Left atrial stimulation Fig. 7 to ro illustrate the effect of electrically induced left atrial extrasystoles. As for right atrium, the $\mathrm{SR}^{-}$is constant ( $90 \mathrm{msec}$ and $100 \mathrm{msec}$ ) but shorter than in right atrial stimulation and varies only when there is an intra-atrial delay (increasing $\mathrm{SP}^{-}$). Unlike right atrial stimulation, $\mathrm{SJ}^{-}$is constant. Similarly, the His deflection, identified only in Case $I$, came after the beginning of the QRS. The $\mathrm{SH}^{-}$ interval seems to be shorter than in right atrial stimulation: $125 \mathrm{msec}$ versus $150 \mathrm{msec}$ for a PS of 550 msec. In Case 2, rapid left atrial stimulation induces the same effects as coupled left atrial stimulation: constant and shorter $\mathrm{SR}^{-}$and constant $\mathrm{SJ}^{-}$(Fig. 6).

In Fig. II and I2, the constancy of $\mathrm{SJ}^{-}$in left atrial stimulation is re-emphasized and contrasted to increasing $\mathrm{SJ}^{-}$in right atrial stimulation. The evolution of $\mathrm{SH}^{-}$is also illustrated.

Retrograde conduction Typical episodes of tachycardia in Case I (Fig. 13) were triggered by an atrial extrasystole at short coupling interval (PS: $200 \mathrm{msec}$ for RA and 340

FIG. 7 Case 1. Coupled left atrial stimulation. To read the ordinate, add Ioo for $\mathrm{SH}^{-}$ and 200 for $\mathrm{SF}^{-}$. Note the constant $\mathrm{SR}^{-}$ measuring 80 msec (shorter than in the right atrium $105 \mathrm{msec}$ ). The $\mathrm{Sf}^{-}$is also constant at 210 msec. Two $\mathrm{PH}^{-}$could be measured at $125 \mathrm{msec}$.

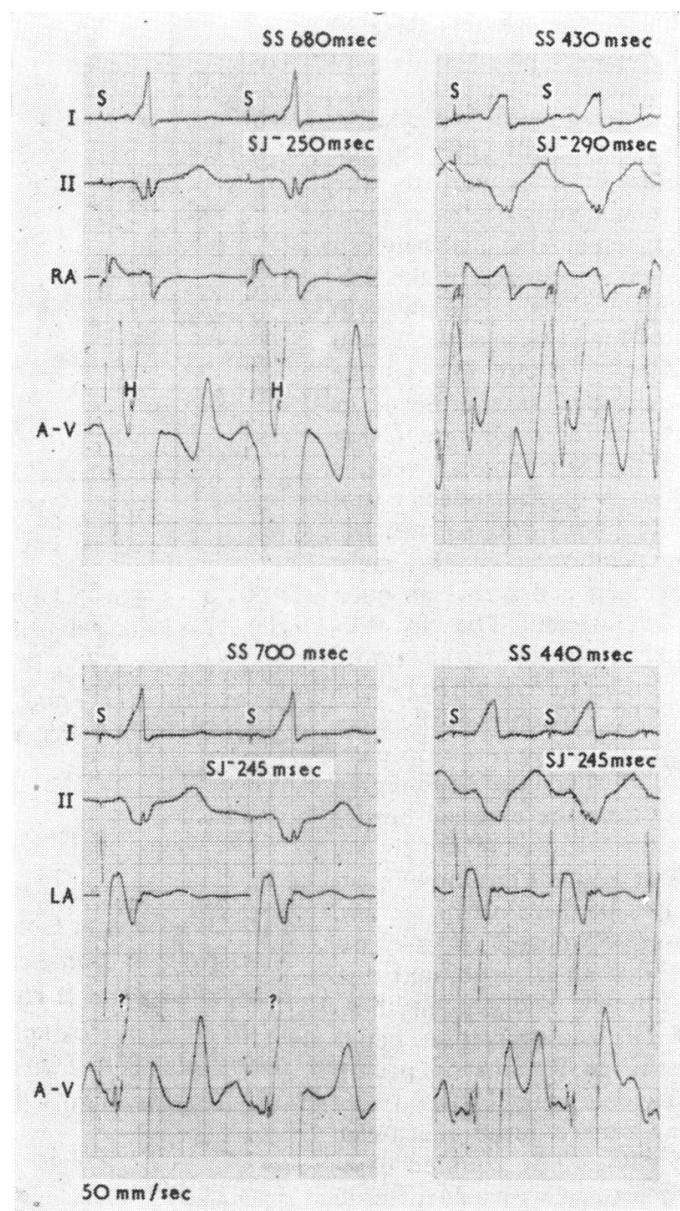

FIG. 6 Case 2. Rapid right atrial and left atrial stimulation. At increasing rate, consider the increasing $\mathrm{Sf}^{-}$interval in right atrium as opposed to constant $\mathrm{SF}^{-}$in the left atrium.

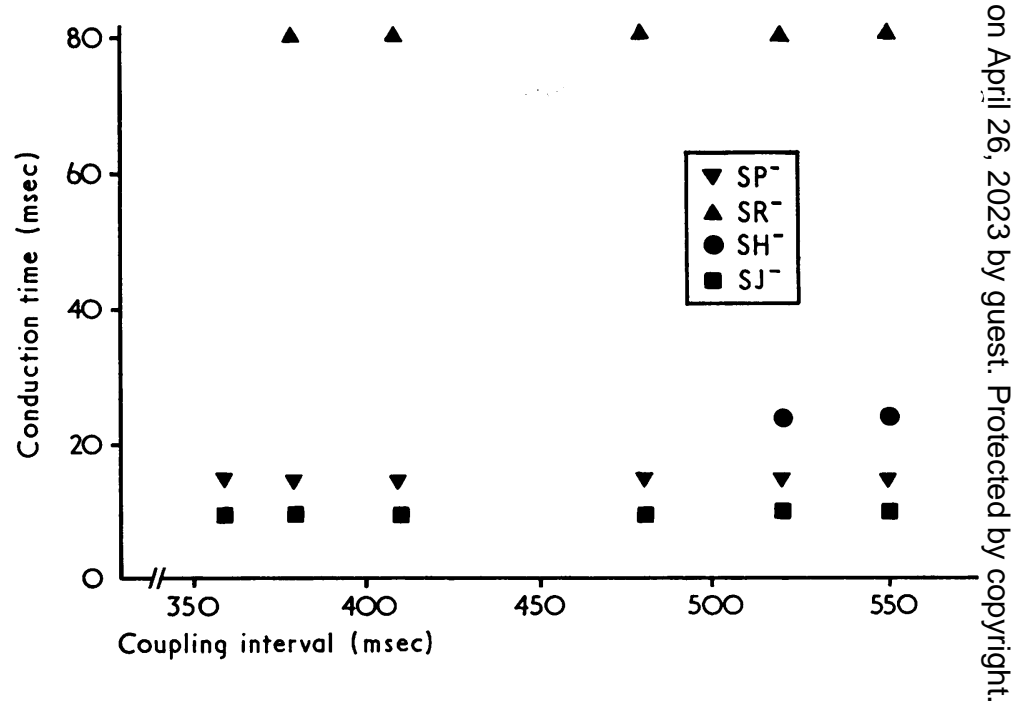


msec for LA). The premature beat is then conducted with a longer PR and is preceded by a His potential. The pre-excitation aspect disappears. This extrasystole initiates a reciprocating tachycardia with normalized QRS and an $\mathrm{H}$ wave in front of each QRS. Moreover the analysis of the retrograde conduction to the atria shows that the depolarization of the high left atrial (HLA) precedes the depolarization of the low right atrial (LRA) at the tricuspid level by $30 \mathrm{msec}$. The same mechanism of tachycardia was noted in Case 2 (Fig. 14). The degree of preactivation of the left atrium is less but real as witnessed by the synchronism of the intrinsicoid deflection recorded in the low right atrium and the high left atrium.

\section{Discussion}

In the WPW syndrome, the normal His bundle activation is short-circuited, as exemplified by a His bundle deflection contemporary with or later than the delta wave. The early activation of the ventricles is, therefore, not dependent on the activation wave coming from this His depolarization. The ventricles are preexcited by an anomalous activation wave coming from a pathway that conducts faster than the normal pathway.

As we shortened the coupling intervals of the right atrial stimulation, the $\mathbf{P R}^{-}$remained constant. The impulsion from the atrium to

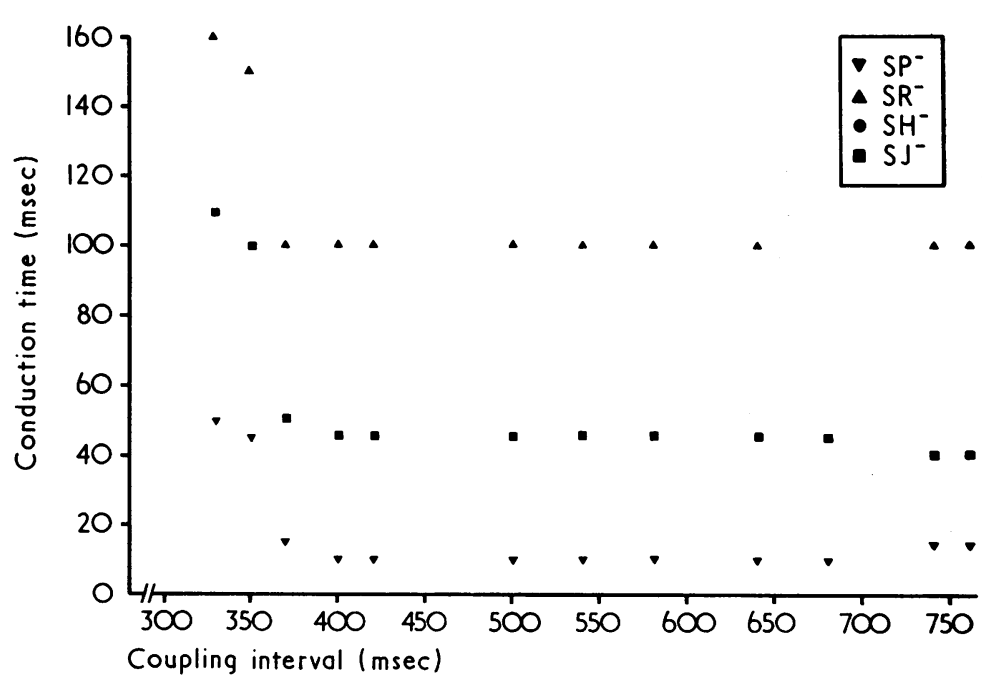

FIG. 8 Case 2. Coupled left atrial stimulation. To read the ordinate, add 200 for SF-. Again note the constancy of the intervals $S R^{-}$ and $\mathrm{Sf}^{-}$. They increase only when there is an intra-atrial delay, at PS $370 \mathrm{msec}$.

the ventricles travelled at a constant speed through the accessory fibres. However, there was an increasing delay in the normal conduction pathways as expected in normal AV nodal conduction. This produced the disappearance of the His bundle deflection into the ventriculogram. Parallel to the increasing

FIG. 9 Case I. Coupled left atrial stimulation. With shorter PS, SF- is constant. His potential $\left(\mathrm{H}^{-}\right)$is visible only in the first two coupled beats. Compare the pre-excitation, the shorter $\mathrm{SR}^{-}$, and the constant Sf- to Fig. 5.
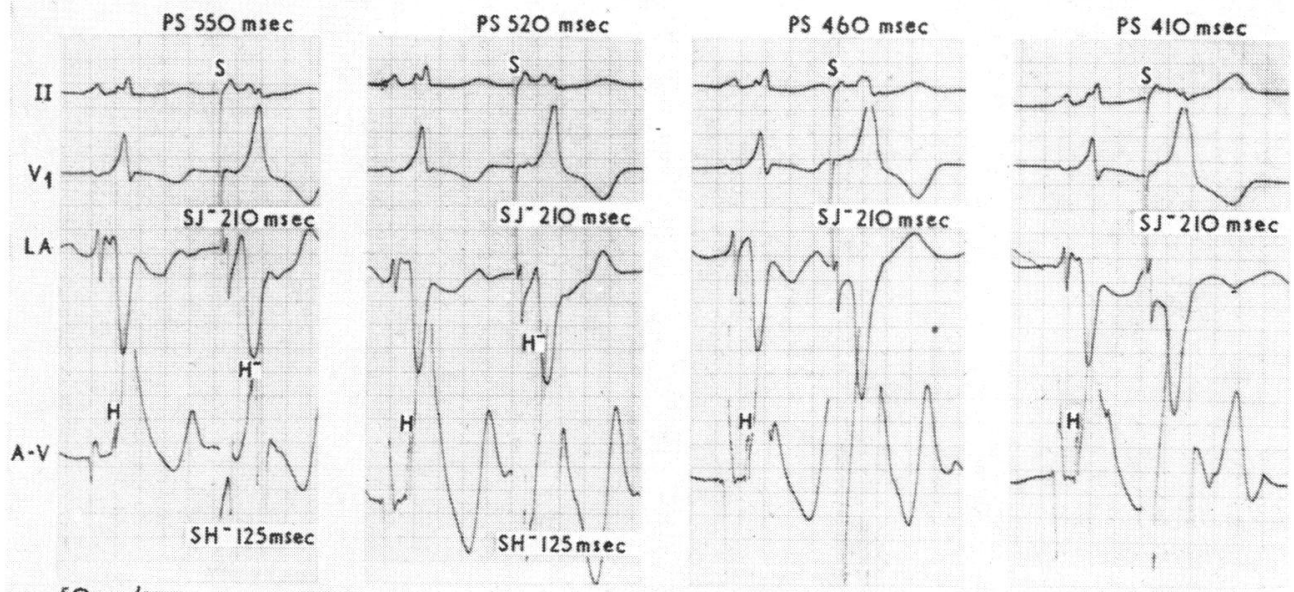

$50 \mathrm{~mm} / \mathrm{sec}$ 


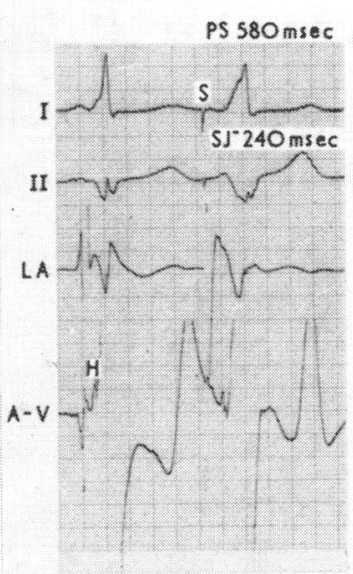

$50 \mathrm{~mm} / \mathrm{sec}$
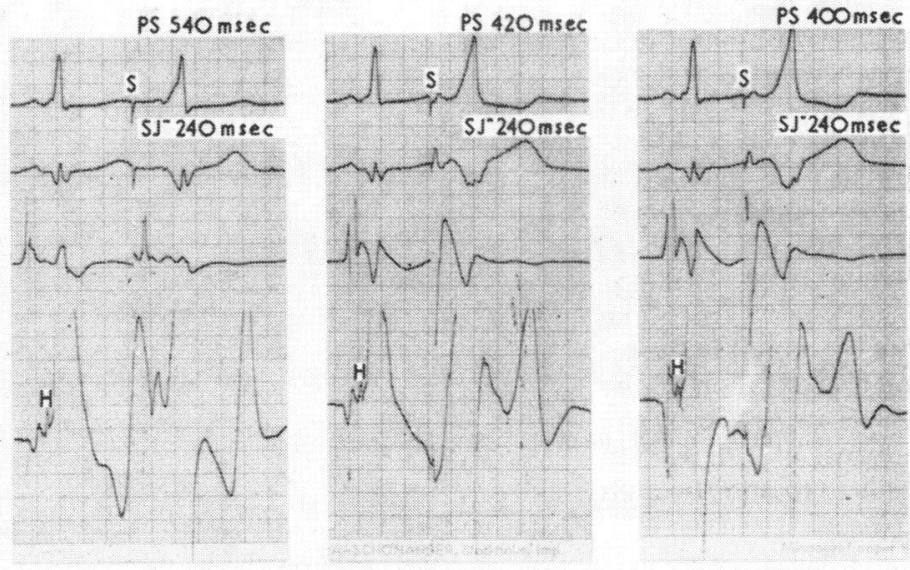

FIG. IO Case 2. Coupled left atrial stimulation. No $\mathrm{H}^{-}$visible in the coupled beats. Note again the short $S R^{-}$and the constant $S f^{-}$.

FIG. II Case I. Comparison of $\mathrm{SF}^{-}$and $\mathrm{SH}^{-}$intervals in the right atrium versus left stimulation. To read the ordinate, add Ioo for $\mathrm{Sf}^{-}$. The increasing $\mathrm{Sf}^{-}$in parallel to $\mathrm{SH}^{-}$ in right atrial stimulation is well delineated as opposed to the constancy of the same intervals in left atrial stimulation.
FIG. I2 Case 2. Comparison of $\mathrm{SF}^{-}$and $\mathrm{SH}^{-}$intervals in the right atrium versus left atrial stimulation. To read the ordinate, add Ioo for $\mathrm{Sf}^{-}$. Increasing $\mathrm{Sf}^{-}$right atrium, constant $S^{-}$in left atrium.
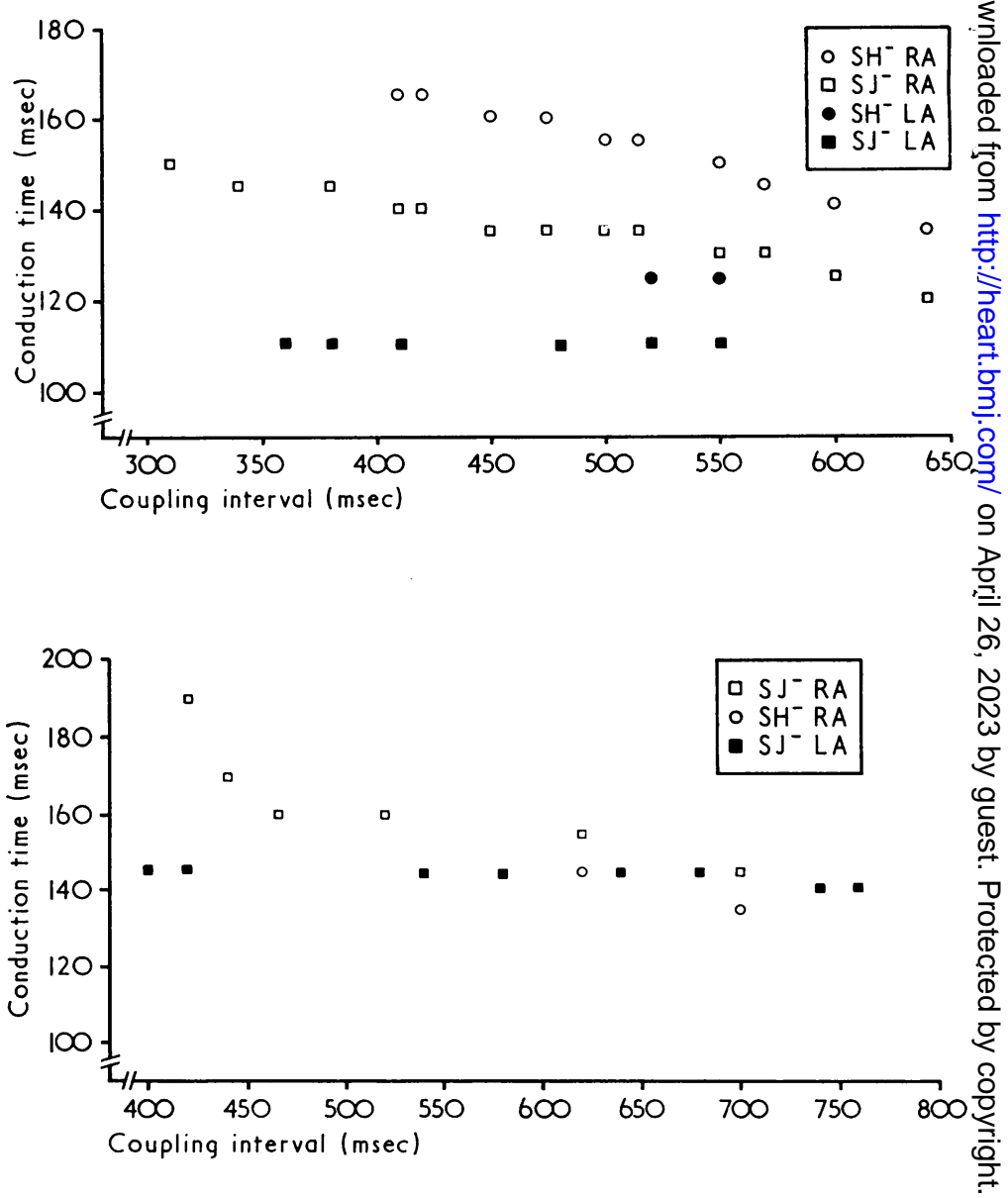
$\mathrm{SH}^{-}$there was also an increasing $\mathrm{SJ}^{-}$. This is due to the fact that the latter part of ventricular activation is dependent on normal $\mathrm{AV}$ conduction. A delay in this conduction will result in a delay in ventricular activation and thus an increasing total depolarization time. The increase in the pre-excitation aspect is explained by the more and more important participation of the accessory conduction pathway to cardiac activation.

In left atrial stimulation, the pre-excitation started earlier as if the stimulation was delivered almost directly to the anomalous conduction fibres. This was responsible for the shorter $\mathrm{SR}^{-}$interval. The $\mathrm{SJ}^{-}$remained constant witnessing that all ventricular depolarization was taken in charge by the excitation coming from the anomalous pathway.

It looks to us as if, at long coupling intervals, the interatrial conduction delay represented by the difference between the SRintervals as measured from right and left atrial excitation leaves sufficient time for normal His bundle conduction to contribute to the ventricular depolarization when the activation originates from the right atrium. This also implies that the stimulus must reach the left atrium to activate the accessory fibres. When the pre-excitation pathway is directly stimulated in the left atrium, there is no time for the impulse travelling through the His bundle to take part in ventricular depolarization. Activation is then totally accomplished by the anomalous pathway.

Another finding is a shorter $\mathrm{SH}^{-}$interval induced by left atrial stimulation in the first patient: the position of the electrodes in the atria is not the cause. A difference in refractory period in the AV node could not be eliminated. A possible explanation is represented by the existence of fibres coming from the anomalous pathway to the AV node (preferential intra-auricular conduction). In fact this pre-excitation of the normal His bundle pathway is difficult to reconcile with an exclusive activation of the ventricles by the accessory pathway. It must be assumed that the impulse coming prematurely to the common bundle is more slowly conducted and then blocked downstream by a refractory state. At shorter coupling intervals $\mathrm{SH}^{-}$lengthens due to the augmentation of the intranodal delay and $\mathrm{H}$ disappears in the QRS.

Regarding the tachycardia, it was our experience, in common with others (Wellens et al., 1971), that the initiation and termination of tachycardia in these two patients were easier from the left cavities particularly from the left atrium. In our two cases, the pattern was the one already described by others

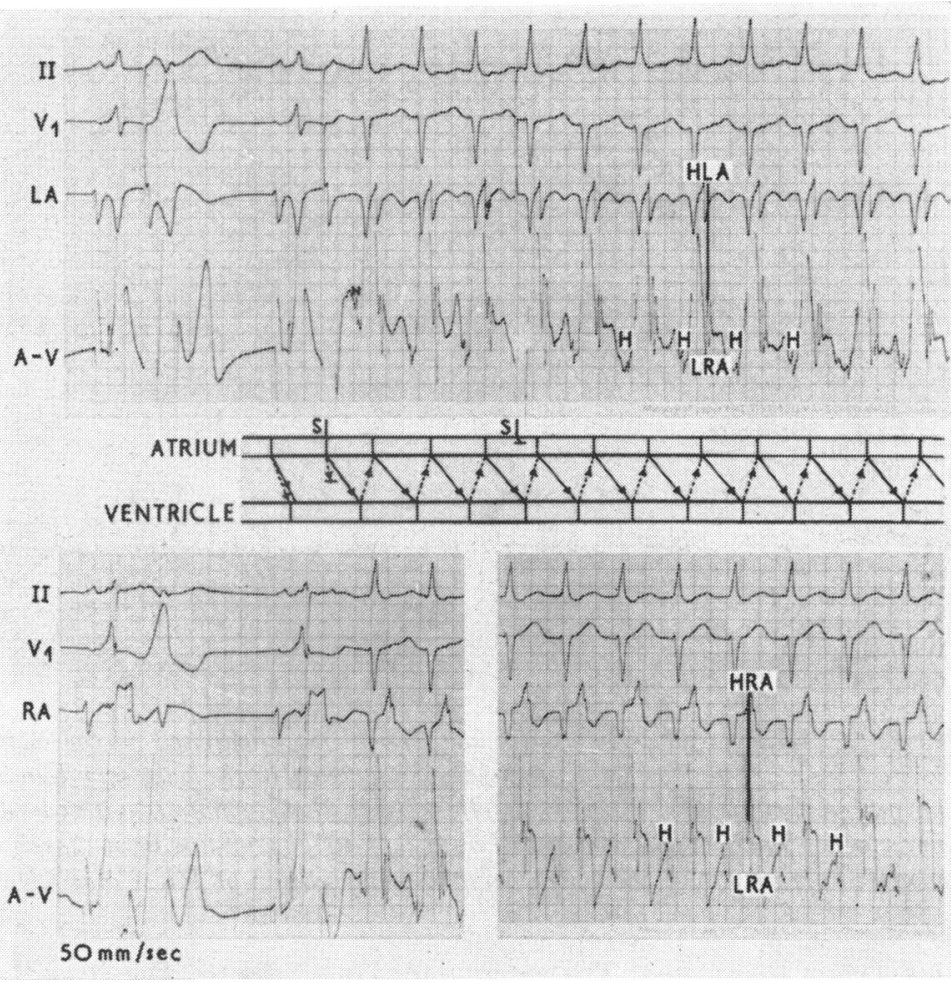

FIG. 13 Case I. The possible mechanism of tachycardia is illustrated under the first tracing. Before the tachycardia, excitation travels in both normal (continuous line) and accessory (interrupted line) pathways. A right atrial stimulus $(S)$ falls in the refractory period of the accessory bundle where it is blocked. However, it is conducted via the normal $A V$ pathway as shown by a longer $P R$ and His deflection in front of the normalized $Q R S$. The re-entry is effected via the anomalous pathway. Anterograde conduction is again via the normal pathway. The circuit is completed and self sustaining. $H L A$ and $H R A$ represent the recording of atrial activity in high left atrium and high right atrium. The LRA is the atrial activity at the tricuspid level. In $H R A$, the atrial activity is recorded after $L R A$, but in HLA depolarization, starts before $L R A$, thus showing a premature retrograde activation of $L A$.

(Castillo and Castellanos, 1970). A premature stimulus finds the accessory pathway in a refractory state and reaches the ventricles via the normal AV fibres (lengthening of PR and disappearance of the pre-excitation). In the meantime, the anomalous bundle has recovered its excitability and allows the return of the ventricular depolarization wave to the atria. Thus tachycardia presenting a circular 


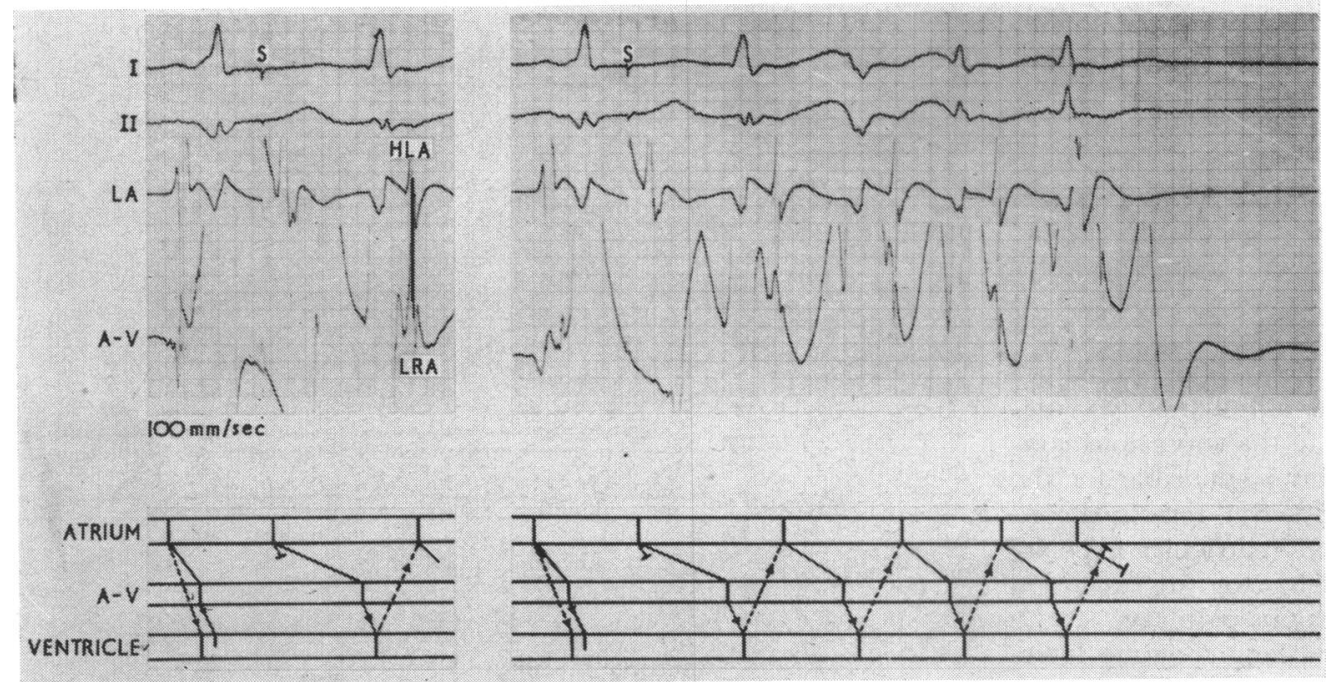

FIG. I4 Case 2. The mechanism of tachycardia illustrated on the right tracing is the same as in Fig. 13: a left atrial stimulus $(S)$ is blocked in the accessory bundle and conducted via the normal $A V$ pathway. Re-entry is effected via the anomalous pathway establishing the circular movement. Tachycardia is stopped by a left atrial stimulus immediately after the fifth ventriculogram. Note the synchronous depolarization of HLA and LRA on the left tracing.

movement is initiated. The anterograde conduction from the atria to the ventricles proceeds through the normal bundle of His system as shown by the His deflection in front of the normalized QRS complexes. Retrograde conduction is probably via the anomalous bundle. In addition, retrograde conduction to the atria was faster to the left than to the right, a fact also demonstrated by Wellens et al. (1971).

The left atrial stimulation showing a shorter $\mathrm{PR}^{-}$, a constant $\mathrm{SJ}^{-}$, the facility of inducing tachycardia in the left cavities, the faster retrograde conduction to the left atrium are all arguments in support of the fact that in the WPW syndrome type $A$ the anomalous pathway lies preferentially on the left side of the heart.

\section{References}

Castellanos, A., Jr., Chapunoff, E., Castillo, C. A., Maytin, O., and Lemberg, L. (1970). His bundle electrograms in two cases of Wolff-ParkinsonWhite (pre-excitation) syndrome. Circulation, 4r, 399.

Castillo, C. A., and Castellanos, A., Jr. (1970). His bundle recordings in patients with reciprocating tachycardias and Wolff-Parkinson-White syndrome. Circulation, 42, 271.

Coumel, Ph., Waynberger, M., Slama, R., and Bouvrain, Y. (1970). Intérêt de l'enregistrement des potentiels hissiens dans la compréhension de certains troubles du rythme. Bloc auriculo-ventriculaire, syndrome de préexcitation, tachycardie jonctionnelle. Archives de Maladies du Coeur et des Vaisseaux, 43, 366.
Durrer, D., Schoo, L., Schuilenburg, R. M., and Wellens, H. J. J. (1967). The role of premature beats in the initiation and termination of supraventricular tachycardia in the Wolff-ParkinsonWhite syndrome. Circulation, 36, 644 .

Lau, S. H., Stein, E., Kosowsky, B. D., Haft, J. I., Lister, J. W., and Damato, A. N. (1967). Atrial pacing and atrio-ventricular conduction in anomalous atrio-ventricular excitation (Wolff-ParkinsonWhite syndrome). American fournal of Cardiology, 19, 354 .

Puech, P., Latour, H., Grolleau, R., and Dufoix, R. (I968). Les stimulations électriques endocavitaires dans l'étude de la préexcitation ventriculaire. Fifth European Congress of Cardiology, Athens, September 1968.

Rosenbaum, F. F., Hecht, H. H. H., and Wilson, F. N. (I945). Potential variations of the thorax and the oesophagus in anomalous atrioventricular excitation (Wolff-Parkinson-White syndrome). American Heart fournal, 29, 281.

Scherlag, B. J., Lau, S. H., Helfant, R. H., Berkowitz, W. D., Stein, E., and Damato, A. N. (1969). Catheter technique for recording $\mathrm{His}$ bundle activity in man. Circulation, 39, 13.

Touboul, P., Clement, C., Roques, J. C., Delahaye, J. P., and Gonin, A. (1971). Le syndrome de WolffParkinson-White. Evidence d'une voie auriculoventriculaire accessoire fournie par l'enregistrement du potentiel du faisceau de His. Archives des Maladies du Coeur et des Vaisseaux, 64, 638.

Wellens, H. J. J., Schuilenberg, R. M., and Durrer, D. (197I). Electrical stimulation of the heart in patients with Wolff-Parkinson-White syndrome, type A. Circulation, 43, 99.

Requests for reprints to Dr. P. Touboul, Service des Soins intensifs, Hôpital Cardio-vasculaire, 59 boulevard Pinel, 69 Lyon $3^{\circ}$, France. 